\title{
Dexmedetomidine facilitates the expression of nNOS in the hippocampus to alleviate surgery-induced neuroinflammation and cognitive dysfunction in aged rats
}

\author{
LIANGYUAN SUO and MINGYU WANG \\ Department of Anesthesiology, Cancer Hospital of China Medical University, \\ Liaoning Cancer Hospital, Shenyang, Liaoning 110042, P.R. China
}

Received May 8, 2020; Accepted April 29, 2021

DOI: $10.3892 /$ etm. 2021.10470

\begin{abstract}
Postoperative cognitive dysfunction (POCD) is a common complication in the postoperative nervous system of elderly patients. Surgery-induced hippocampal neuroinflammation is closely associated with POCD. Dexmedetomidine (DEX) is an effective $\alpha 2$-adrenergic receptor agonist, which can reduce inflammation and has neuroprotective effects, thereby improving postoperative cognitive dysfunction. However, the mechanism by which DEX improves POCD is currently unclear. The purpose of the present study was therefore to identify how DEX acted on POCD. Male Sprague Dawley rats with exposed carotid arteries were used to mimic POCD. Locomotor activity was accessed by the open field test and the Morris water maze was performed to estimate spatial learning, memory and cognitive flexibility. Following animal sacrifice, the hippocampus was collected and cell apoptosis was determined by terminal dexynucleotidyl transferase (TdT)-mediated dUTP nick end labeling staining. Subsequently, the expression of apoptosis-related proteins Bax, Bcl-2, cleaved caspase-3 and cleaved caspase- 9 was determined by western blotting and the concentrations of TNF- $\alpha$, IL- 6 , IL-1 $\beta$ and IL-10 were measured in serum using ELISA. Nitric oxide synthase and neuronal nitric oxide synthase activities in the hippocampus were also measured. The T lymphocyte subsets were analyzed by flow cytometry to evaluate the immune function in each
\end{abstract}

Correspondence to: Dr Mingyu Wang, Department of Anesthesiology, Cancer Hospital of China Medical University, Liaoning Cancer Hospital, 44 Xiaoheyan Road, Dadong, Shenyang, Liaoning 110042, P.R. China

E-mail: mingyuwang1211@163.com

Abbreviations: POCD, postoperative cognitive dysfunction; DEX, dexmedetomidine; TUNEL, terminal dexynucleotidyl transferase (TdT)-mediated dUTP nick end labeling; OFT, open field test; MWM, morris water maze; NOS, nitric oxide synthase; nNOS, neuronal nitric oxide synthase; L-NAME, NG-nitro-L-arginine methyl ester

Key words: cognitive dysfunction, dexmedetomidine, immune function loss, neuroinflammation, postoperative group. Compared with the surgery group, DEX ameliorated POCD by improving cognitive dysfunctions and immune function loss, and attenuated neuroinflammation and neuronal apoptosis.

\section{Introduction}

Postoperative cognitive dysfunction (POCD) is a common clinical syndrome in elderly patients following surgery $(1,2)$ which negatively affects quality of life and is associated with high mortality $(3,4)$. The pathogenesis of POCD is not fully understood, but has been determined to involve neuroinflammation, oxidative stress, autophagy disorder, impaired synaptic function and a lack of neuro-nutritional support (5). Several studies in animals have found that neuroinflammation might be the crucial factor in POCD (6-8). Currently, the treatment of POCD is inconclusive, therefore, the modifiable factors of POCD should be determined and preventive strategies must be formulated.

Dexmedetomidine (DEX) is an effective $\alpha 2$-adrenergic receptor agonist (9). DEX has been widely reported in ischemic-reperfusion models, exhibiting resistance in free radicals and cell apoptosis $(10,11)$. Previous studies have demonstrated that DEX can reduce inflammation and has neuroprotective effects, thereby improving postoperative cognitive dysfunctions $(12,13)$. Further research confirmed that the use of DEX during carotid endarterectomy can reduce the incidence of POCD after surgery short term, which is associated with the inhibition of the inflammatory response and an increase in the expression of brain-derived neurotrophic factor (14). However, the specific mechanism by which DEX improves POCD remains unclear.

Neuronal nitric oxide synthase (nNOS) is a constitutive neuronal enzyme that is important in regulating central nervous system functions (15). Previous reports have confirmed that inhibition of nNOS can impair learning and memory (16). In addition, DEX can serve a protective role in brain injury by inhibiting nNOS-nitric oxide signaling (17). Treatment with DEX can also alleviate traumatic brain injury and promote cognitive and motor recovery after brain injury (18). Thus, to determine the association between DEX and nNOS in POCD, NG-nitro-L-arginine methyl ester (L-NAME), a nonspecific 
NOS inhibitor, was used in the present study. The carotid artery of aged rats was exposed to mimic POCD and the expression of relevant indicators following surgery was investigated, with or without L-NAME treatment.

\section{Materials and methods}

Animals. 50 Male Sprague Dawley rats (weight, 500-650 g; age, 20 months old) were purchased from Charles River Laboratories and housed in groups under controlled environmental conditions. All animals were grouped in a $12 \mathrm{~h}$ light/dark cycle in a room with controlled temperature and humidity $\left(22 \pm 1^{\circ} \mathrm{C}\right.$ and $\left.50-60 \%\right)$ and fed water and food ad libitum. All experiments were approved by the Animal Experiment Center of the Institute of Radiation Medicine of the Chinese Academy of Medical Sciences.Study design. Rats were randomly divided into 5 groups: i) Sham; ii) surgery; ii) surgery + L-NAME; iv) surgery + DEX; and v) surgery + $\mathrm{DEX}+\mathrm{L}-\mathrm{NAME}$, with 10 rats per group. At $30 \mathrm{~min}$ prior to surgery, rats in each group received the following treatment: i) Rats in the sham group did not receive any treatment; ii) rats in the surgery + L-NAME group were injected intraperitoneally with $25 \mathrm{mg} / \mathrm{kg}$ L-NAME; iii) rats in the surgery + DEX group were injected intraperitoneally with $12 \mu \mathrm{g} / \mathrm{kg} \mathrm{DEX}$; iv) rats in the surgery + L-NAME group were injected with $25 \mathrm{mg} / \mathrm{kg}$ L-NAME; and v) rats in the surgery + DEX + L-NAME group were injected intraperitoneally with $12 \mu \mathrm{g} / \mathrm{kg}$ DEX and $25 \mathrm{mg} / \mathrm{kg} \mathrm{L-NAME}$. The open field test (OFT) was performed on days 8 and 16. The Morris water maze (MWM) training was conducted on days 9-13 and days 17-21, and the MWM test was conducted on days 14 and $2 \mathrm{ml}$ tail vein blood was collected both prior to surgery and on day 4 and 9 following surgery. Surgical methods were based on previous reports (5). Firstly, following anesthetization by intraperitoneal injection of $3 \%$ sodium pentobarbital $(50 \mathrm{mg} / \mathrm{kg})$, a $1.5 \mathrm{~cm}$ opening was cut at the midline of the neck, opening the soft tissue on the trachea. Subsequently, a $1 \mathrm{~cm}$ long part of the right common carotid artery was removed and separated from the adjacent tissue. The skin was sutured and surgery was conducted in a sterile environment. Once tail vein blood $(2 \mathrm{ml})$ had been collected 9 days post surgery, the rats were anesthetized with $3 \%$ sodium pentobarbital $(50 \mathrm{mg} / \mathrm{kg})$ and treated with cardiac perfusion. Rats were euthanized by intraperitoneal injection with excessive sodium pentobarbital $(200 \mathrm{mg} / \mathrm{kg})$ and the vena cava blood and the hippocampus tissue were collected for ELISA, western blotting, terminal dexynucleotidyl transferase (TdT)-mediated dUTP nick end labeling (TUNEL) staining and immunohistochemical staining (Fig. 1).

OFT. Animal locomotor activity was monitored for $10 \mathrm{~min}$ using an OFT. The test was conducted on days 8 and 16. A wooden box $(100 \times 100 \times 45 \mathrm{~cm})$ was divided into 16 squares and the rats were placed in the center of the box. Each rat was placed in the corner to acclimate for $5 \mathrm{~min}$, and then placed into a new square. While the rat crossed the square, a vertical lattice counting and horizontal lattice counting was used to monitor and record movements over a 5 min period. After each rat was tested, the wooden box was cleaned with $75 \%$ ethanol and wiped dry with cotton balls to minimize the effect of odor on subsequent experiments.
MWM test. The MWM test was used to assess spatial learning, memory and cognitive flexibility in each group. The maze consisted of orientation, navigation and spatial probe tests. A swimming pool with a diameter of $122 \mathrm{~cm}$ and a depth of $35 \mathrm{~cm}$ was divided into four equal parts. The water in the swimming pool, with a depth of $17 \mathrm{~cm}$, was heated to $22^{\circ} \mathrm{C}$ prior to the experiment. A platform $\left(10 \mathrm{~cm}^{2}\right)$ was invisibly located in the center of the target quadrant. During the experiment, the hidden platform was placed in 1 of the quadrants $1.5 \mathrm{~cm}$ below the water surface. Specific methods were based on a previous report (19).

TUNEL staining. Hippocampus tissue were fixed with $4 \%$ paraformaldehyde for $24 \mathrm{~h}$ at room temperature (RT), dehydrated using graded ethanol, embedded in paraffin and sliced at a thickness of $5 \mu \mathrm{m}$. Apoptotic cell death in hippocampus tissue was detected by utilizing a TUNEL kit (cat. no. 11684795910; Roche Diagnostics) according to the manufacturer's instructions. Slices were stained using the solution included in the TUNEL kit at RT for $1 \mathrm{~h}$, and then stained using Hematoxylin at RT for $\sim 3$ min. After sealing with neutral gum sections were imaged and captured using light microscopy (magnification, $x 40$ ). Nine fields of view were observed in each group and analyzed using 1.0 ImageJ software (National Institutes of Health).

Western blot analysis. At 4 days after surgery, proteins were extracted from hippocampus tissue using RIPA lysis buffer (Applygen Technologies, Inc.) and a mixture of protease inhibitors and phosphatase inhibitors (Pierce; Thermo Fisher Scientific, Inc.). Extracted protein was measured using a BCA kit (Nanjing Jiancheng Bioengineering Institute) and mixed with $5 \mathrm{X}$ loading buffer. Samples (40 $\mu \mathrm{g} / \mathrm{lane})$ were separated using a $12 \%(\mathrm{w} / \mathrm{v})$ gradient SDS gel and transferred to PVDF membranes. After blocking with 5\% skimmed milk at RT for $90 \mathrm{~min}$, the blots were incubated with the following primary antibodies at $4^{\circ} \mathrm{C}$ overnight: $\operatorname{Bax}(1: 2,000$; cat. no. ab32503; Abcam), Bcl-2 (1:2,000; cat. no. ab196495; Abcam), cleaved caspase-3 (1:1,500; cat. no. ab32042; Abcam), cleaved caspase-9 (1:2,000; cat. no. ab2324; Abcam) and GAPDH (1:1,000; cat. no. ab199553; Abcam). The membranes were then incubated with goat anti-rabbit HRP conjugated secondary antibodies [1:10,000; cat. no. 70-GAR0072; Multi Sciences (Lianke) Biotech Co., Ltd.] at RT and washed with TBST (Tris- $\mathrm{HCl}$ buffer and $1 \%$ Tween). Bands were then detected using an ECL kit (Bio-Rad Laboratories, Inc.) and quantified using Image Lab 3.0 software (Bio-Rad).

ELISA. Blood was collected prior to and at 4, 9 days after surgery and centrifuged at 4,000 $\mathrm{x}$ g for $10 \mathrm{~min}$ at $4^{\circ} \mathrm{C}$ to prepare the serum. Concentrations of TNF- $\alpha$ (cat. no. CSB-E11987r; Cusabio Technology), IL-6 (cat. no. CSB-E04640r; Cusabio Technology), IL-1 $\beta$ (cat. no. CSB-E08055r; Cusabio Technology) and IL-10 (cat. no. CSB-E04595r; Cusabio Technology) in serum were determined using ELISA kits following the manufacturer's instructions.

NOS activity detection in hippocampus The expression of NOS in the hippocampus was detected according to a previous report (20). After protein extraction that was performed as described above, NOS activity was measured using the NO 


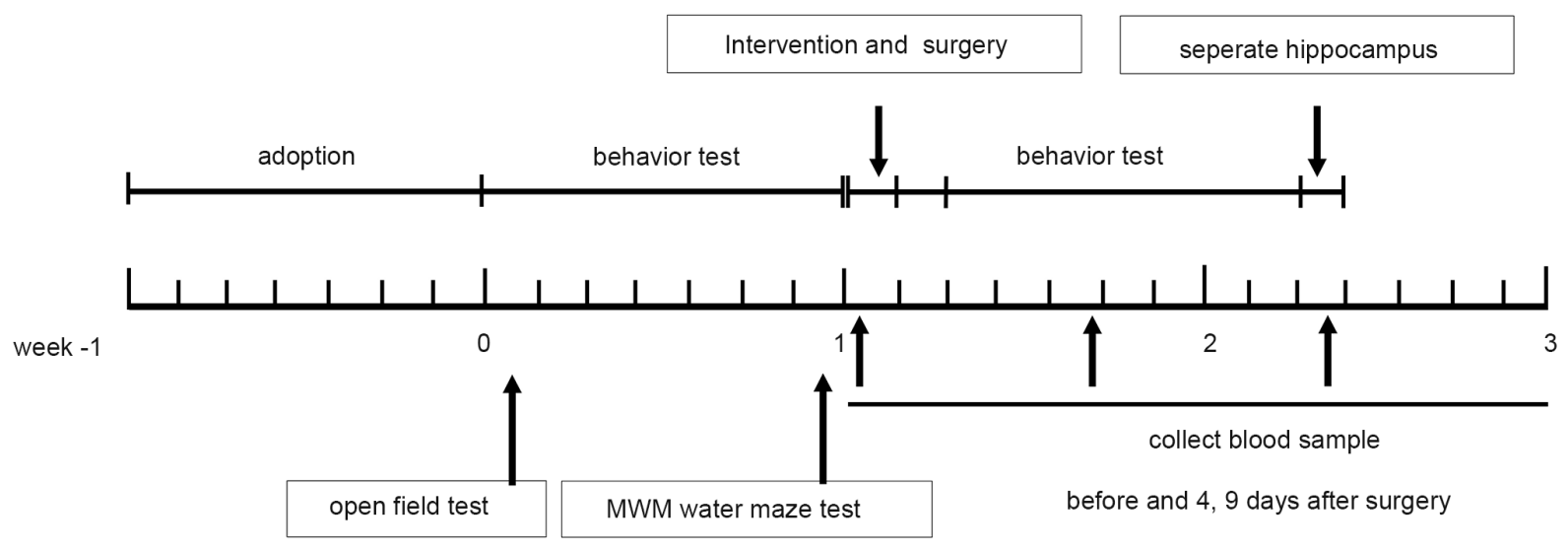

Figure 1. Establishment and sampling procedure of the rat model induced by surgery.

Fluoro-metric Assay kit (Nanjing Jiancheng Bioengineering Institute) in accordance with the manufacturer's protocol. NOS activity was then detected by measuring absorbance at $550 \mathrm{~nm}$ and calculated using the standard curve.

Immunohistochemical staining. Hippocampus tissue was pretreated according to the protocol described in the TUNEL staining paragraph. Immunohistochemical staining was performed using de-paraffinized sections. Briefly, $5 \mu \mathrm{m}$-sections were preheated in an oven, de-paraffinized by xylene and rehydrated via graded ethanol. The sections were then incubated with anti-nNOS (1:200; cat. no. ab5586; Abcam) primary antibodies at RT for 90 min followed by HRP-conjugated goat anti-rabbit secondary antibodies (1:5,000, cat. no. ab205718; Abcam) incubation at $37^{\circ} \mathrm{C}$ for $90 \mathrm{~min}$. A DAB kit (cat. no. ZLI-9018; OriGene Technologies, Inc.) was used to visualize the sections with a microscope. Nuclei were stained using hematoxylin at RT for $3 \mathrm{~min}$ and the images were captured using a light microscope (magnification, x20).

Flow cytometry. Flow cytometry was used to detect blood Tlymphocyte subsets (CytomicsFC500; Beckman Coulter,Inc.). Blood samples with the heparin anticoagulant were collected at various times according to the experimental design. Specimens were prepared using FACS lysing solution (BD Biosciences) at RT for $10 \mathrm{~min}$. Cells were then permeabilized and fixed with cytofix/Cytoperm Plus solution (BD Biosciences) at RT for $20 \mathrm{~min}$. Cells were labeled with the following monoclonal antibodies conjugated with different fluorescent dyes at $4^{\circ} \mathrm{C}$ for $30 \mathrm{~min}$ : Anti-CD3-PE (5 $\mu 1 / 10^{5}$ cell; cat. no. 15-0038-42; Invitrogen; Thermo Fisher Scientific, Inc.), anti-CD4-APC (5 $\mu 1 / 10^{5}$ cell; cat. no. 17-0049-42; Invitrogen; Thermo Fisher Scientific, Inc.) and anti-CD8-FITC $\left(0.1 \mu \mathrm{g} / 1 \times 10^{6}\right.$ cells; cat. no. MA5-17604; Invitrogen; Thermo Fisher Scientific, Inc.). Finally cells were analyzed using a FACS CANTO ${ }^{\text {TM }}$ II flow cytometer (Becton-Dickinson) and data were analyzed using Flowjo 7.6.1 software (Tree Star, Inc.).

Statistical analysis. All experiments were repeated three times and the final data were expressed as the mean \pm SD. Statistically significant differences were analyzed using one-way ANOVA analysis and Tukey multiple comparison tests. A value of $\mathrm{P}<0.05$ was considered statistically significant. All statistical analyses were conducted using GraphPad Prism 7.0 (GraphPad Software, Inc.).

\section{Results}

Comparison of the behavioral differences of rats in each group. The locomotor activity of rats was initially measured using the OFT. After evaluating both horizontal lattice counts and vertical lattice counts, the results demonstrated that there was no significant difference between the rats in each group (Fig. 2A), indicating that the observed cognitive deficits of the OFT test did not affect locomotor capacity. The MWM test was performed to investigate spatial learning, memory and cognitive flexibility in each group of rats. Cognitive and behavioral tests in the surgery group revealed an impaired exploratory behavior as indicated by significant increases in the escape latency, total distance of swimming and decrease in the times of platform crossing, when compared with the corresponding group prior to surgery (Fig. 2B). Regarding escape latency and total distance of swimming, the surgery + DEX group demonstrated significant decreases when compared with the surgery group, while demonstrating significantly increased times in crossing platforms. Compared with the surgery + DEX and surgery + L-NAME groups, the escape latency and total swimming distance of the surgery + DEX + L-NAME group were significantly increased. In addition, the escape latency and total swimming distance in the surgery + DEX + L-NAME group were increased compared with the surgery + DEX group. The results of swimming speed indicated that there was no statistical difference between the groups. However, the time of crossing platform in the surgery + DEX + L-NAME group was significantly increased compared with the surgery + L-NAME group, but was remarkably decreased compared with the surgery + DEX group. The results indicated that cognitive impairment did not affect the locomotor activity of rats and that DEX improved the cognitive impairment of rats caused by surgery.

Comparison of apoptosis in hippocampus tissue. The results of TUNEL staining are indicated in Fig. 3. Significant neuronal apoptosis was identified in the hippocampus of the sham group when compared with the hippocampus of operated rats. CA1, CA3 and DG are the dentate gyrus that comprise the main 
A

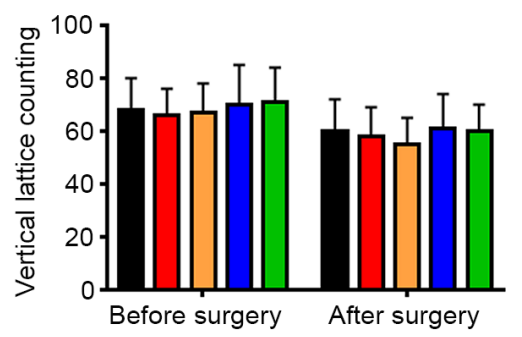

B

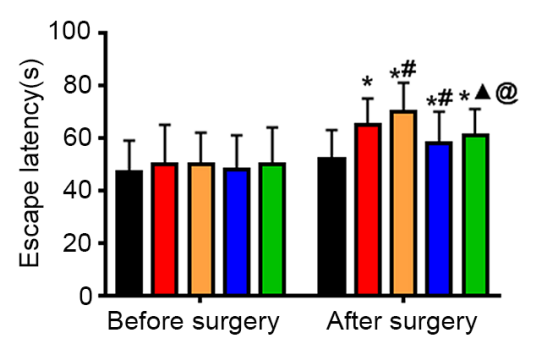

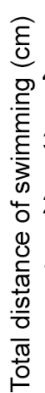

sham

$\square$ surgery

$\square$ surgery+L-NAME

$\square$ surgery+Dex

$\square$ surgery+Dex+L-NAME
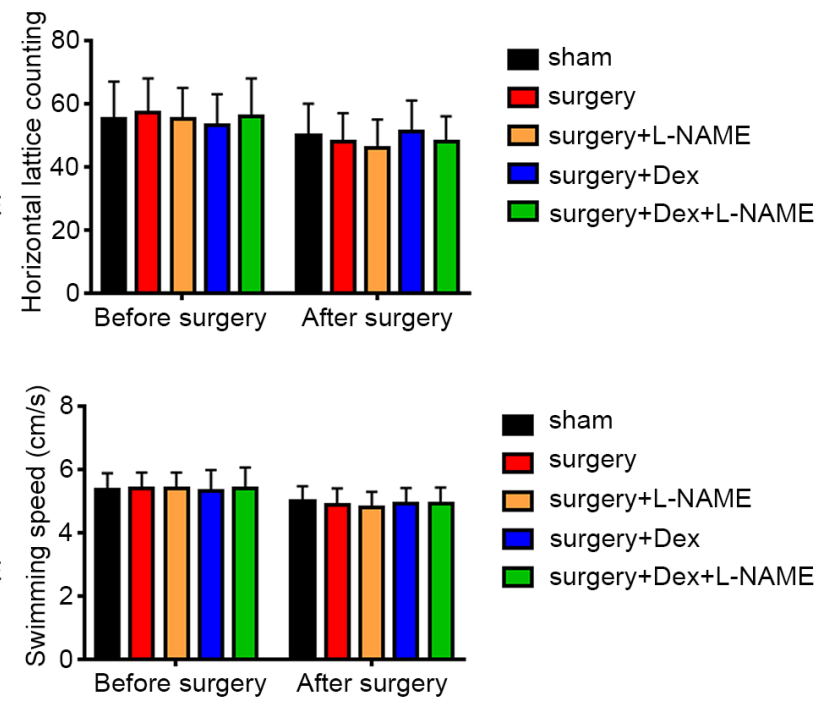

Figure 2. Behavioral differences of rats in each group. (A) Vertical lattice counting and horizontal lattice counting in the open field test. (B) Escape latency, swimming speed, total distance of swimming and times of crossing platform in the Morris water maze test before and after surgery. Data are expressed as the mean $\pm \mathrm{SD}(\mathrm{n}=10)$. ${ }^{*} \mathrm{P}<0.05$ vs. sham; ${ }^{\#} \mathrm{P}<0.05$ vs. the surgery group; ${ }^{\wedge} \mathrm{P}<0.05$ vs. the surgery $+\mathrm{L}-\mathrm{NAME}$ group; ${ }^{\circledR} \mathrm{P}<0.05$ vs. the surgery $+\mathrm{DEX}$ group. L-NAME, G-nitro-L-arginine methyl ester; DEX, dexmedetomidine.

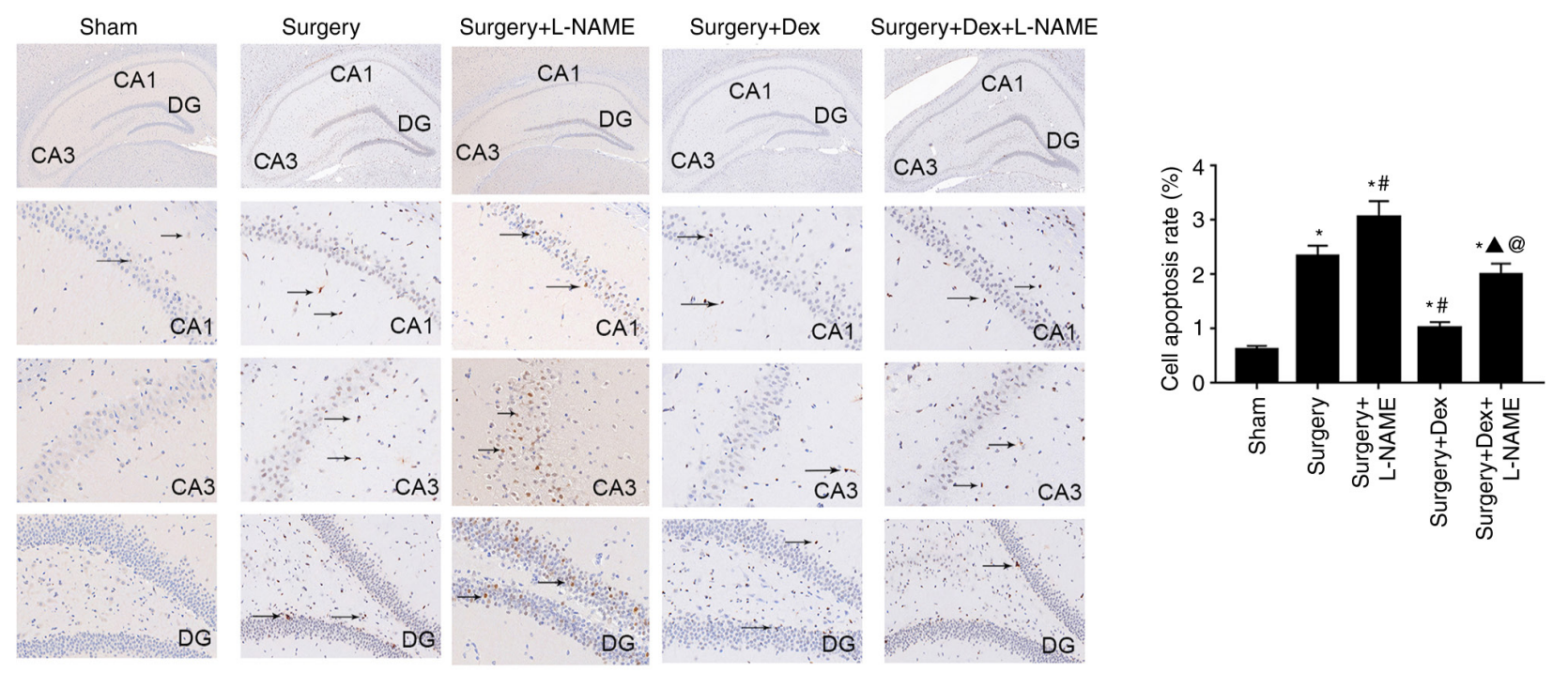

Figure 3. Apoptosis rate of hippocampal neurons was determined by TUNEL staining. Data are expressed as the mean \pm SD ( $=10$ ). CA1, CA3 and DG are the dentate gyrus that comprise the main hippocampus. The arrows in the figure indicate the TUNEL positive area. ${ }^{*} \mathrm{P}<0.05$ vs. sham; ${ }^{\#} \mathrm{P}<0.05$ vs. the surgery group; ${ }^{\wedge} \mathrm{P}<0.05$ vs. the surgery + L-NAME group; ${ }^{\circledR} \mathrm{P}<0.05$ vs. the surgery + DEX group. L-NAME, G-nitro-L-arginine methyl ester; DEX, dexmedetomidine.

hippocampus. There was ameliorated apoptosis in the hippocampus tissue when the rats were treated with DEX or DEX + L-NAME following surgery. Moreover, rats in the surgery + DEX group presented a significantly decreased cell apoptosis rate compared with the surgery + DEX + L-NAME and the surgery + L-NAME groups. To further evaluate cell apoptosis in hippocampus tissue, apoptosis-associated proteins were detected by western blotting (Fig. 4A). The results indicated that the expression levels of Bax, cleaved caspase- 3 and cleaved caspase- 9 of the surgery group were significantly increased 
A

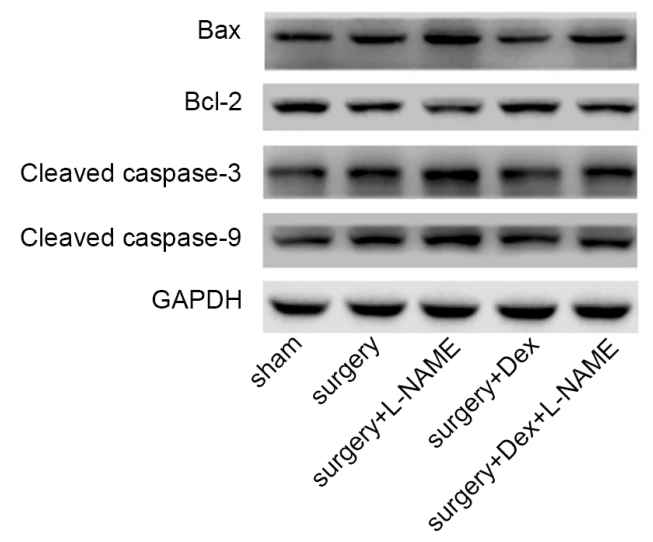

B

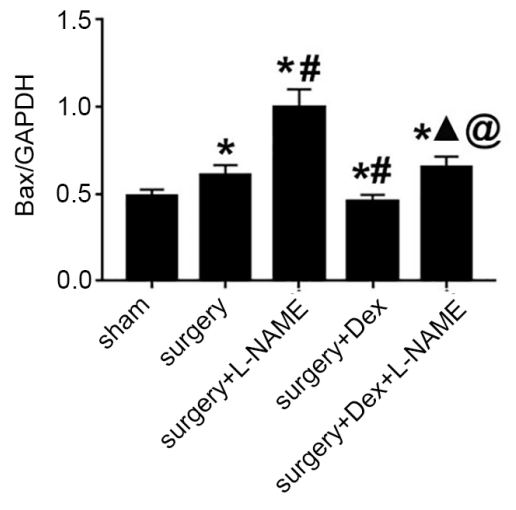

C

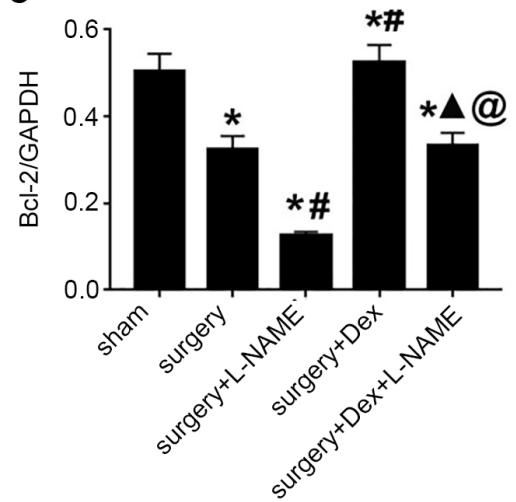

D

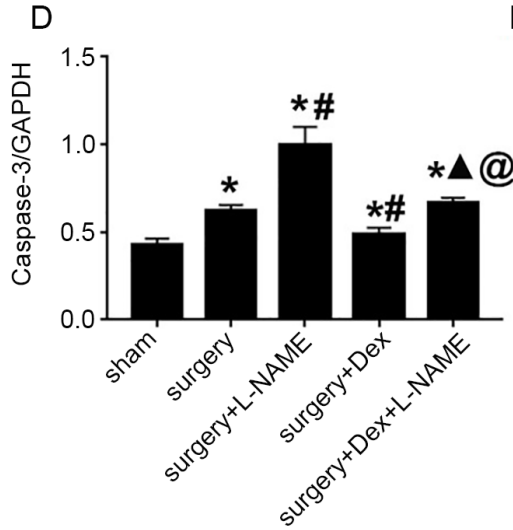

E

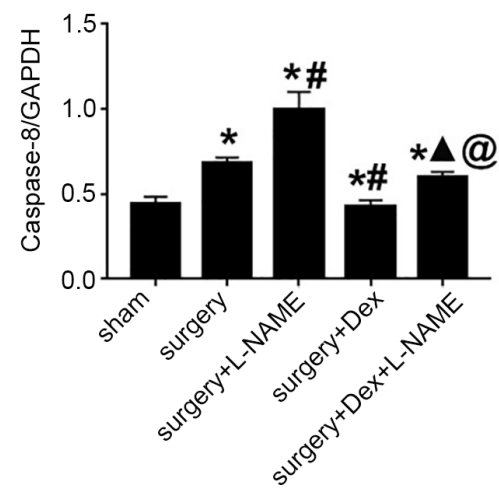

Figure 4. Levels of apoptosis factors Bax, Bcl-2, cleaved caspase-3 and cleaved caspase-9 in the hippocampus were determined by western blotting. (A) Western blot analysis demonstrating the expression of apoptosis factors. (B) The levels of Bax/GAPDH in each group. (C) The levels of Bcl-2/GAPDH in each group. (D) The levels of cleaved caspase-3/GAPDH in each group. (E) The levels of cleaved caspase-9/GAPDH in each group. Data are expressed as the mean $\pm \mathrm{SD}(\mathrm{n}=10)$. ${ }^{*} \mathrm{P}<0.05$ vs. sham; ${ }^{\#} \mathrm{P}<0.05$ vs. the surgery group; ${ }^{\wedge} \mathrm{P}<0.05$ vs. the surgery $+\mathrm{L}-\mathrm{NAME}$ group; ${ }^{\circledR} \mathrm{P}<0.05$ vs. the surgery $+\mathrm{DEX}$ group. L-NAME, G-nitro-L-arginine methyl ester; DEX, dexmedetomidine.

compared with the sham group. Compared with surgery group, rats treated with DEX and surgery significantly attenuated the upregulation of Bax, cleaved caspase- 3 and cleaved caspase- 9 levels. The surgery + DEX + L-NAME group demonstrated the inhibition in the expression changes of Bax, cleaved caspase-3 and cleaved caspase-9, when compared with the surgery + L-NAME group. However, Bax, cleaved caspase-3 and cleaved caspase-9 expression in the surgery + DEX + L-NAME group exceeded that of the surgery + DEX group (Fig. 4B, D and E). In addition, the surgery + DEX group demonstrated an upregulated expression of $\mathrm{Bcl}-2$ (Fig. 4C). Together, these findings indicated that DEX significantly attenuated apoptosis induced by surgery.

Increase of inflammatory cytokines. Inflammatory cytokine plasma concentration levels indicate an apparent systemic inflammatory response, which serves critical roles in cognitive function (21). The release of proinflammatory cytokines TNF- $\alpha$, IL- 6 and IL-1 $\beta$, and anti-inflammatory cytokines IL-10 were therefore measured at 4 and 9 days after surgery. As demonstrated in Fig. 5A, all surgery groups had higher systemic inflammatory cytokine levels than those of the sham group at 4 days after surgery. Compared with the surgery + DEX group, the levels of proinflammatory and anti-inflammatory cytokines in the surgery + DEX + L-NAME group were statistically significant. Furthermore, compared with the surgery + L-NAME group, the surgery + DEX + L-NAME group demonstrated a decreased tendency in the expression of proinflammatory cytokines and an increased trend in the expression of anti-inflammatory cytokines. Furthermore, the expressions of proinflammatory cytokines and anti-inflammatory cytokines in the surgery + DEX group on days 4 and 9 after surgery were close to the baseline.

Comparison of NOS and $n N O S$ in rats. After evaluating the total NOS activity in hippocampus tissue, a significant decline in the surgery group was detected when compared with the sham group (Fig. 5B). The surgery + DEX and surgery + DEX + L-NAME groups were significantly lower than the sham group (Fig. 5B) and DEX treatment significantly increased the total NOS activity, as evidenced by the higher total NOS activity in the surgery + DEX group compared with the surgery group. Fig. 5C indicated that nNOS-positive neurons were widely distributed in the sham group tissues. After surgery, the nNOS-positive neurons indicated a significant reduction; the same was also exhibited in the surgery + L-NAME group compared with the sham group. Compared with the surgery group, post-surgery rats treated with DEX exhibited an increase in the nNOS-positive neurons. However, the surgery + DEX + L-NAME group demonstrated a significantly decreased nNOS level compared with the surgery + DEX group, which was still higher that of the surgery + L-NAME group (Fig. 5C).

Comparison of the T lymphocyte subsets. Among the $\mathrm{T}$ lymphocyte subsets, $\mathrm{CD}^{+}$samples included all mature 
A
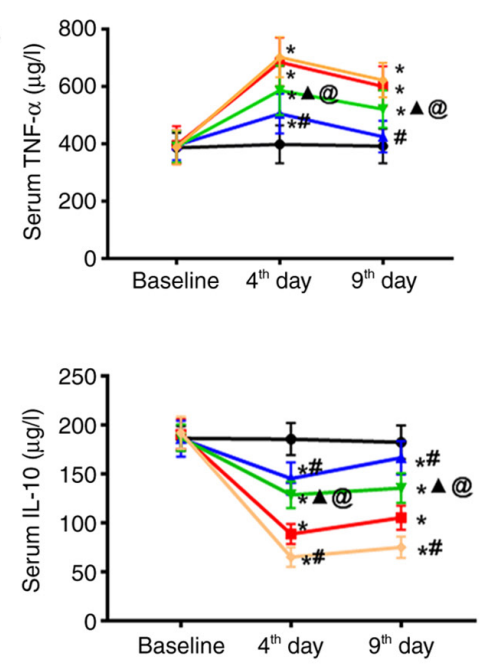

$\rightarrow$ Sham

- Surgery

$\rightarrow$ Surgery+L-NAME

$\mp$ Surgery+Dex

* Surgery+Dex+L-NAME

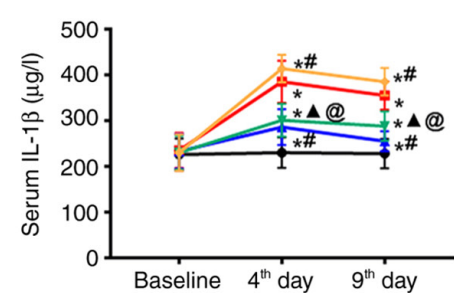

$\mathrm{B}$

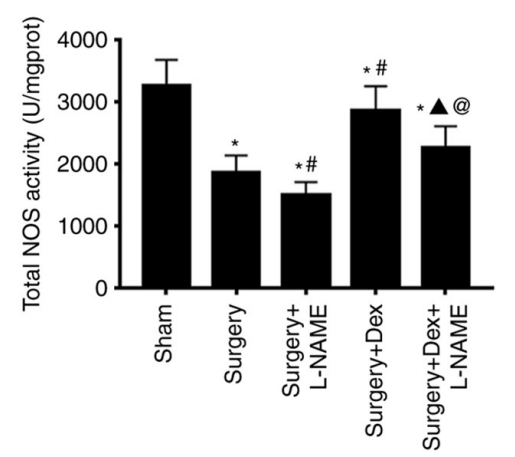

C

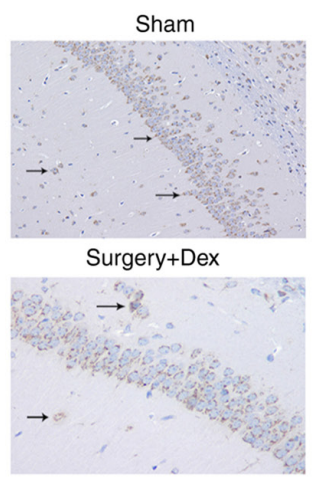

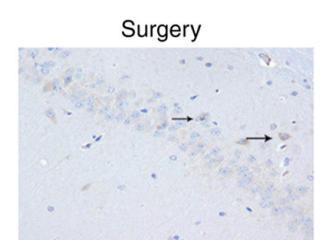

Surgery+Dex+L-NAME

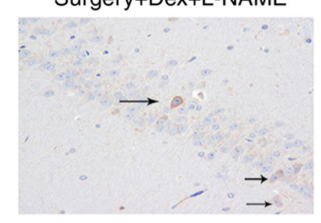

Surgery+L-NAME
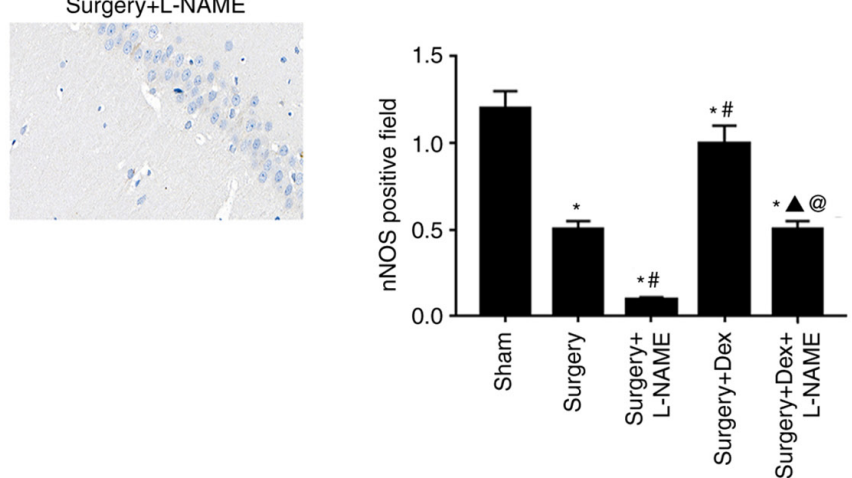

Figure 5. Comparison of serum levels of inflammatory cytokines, NOS and nNOS expressions in the hippocampus from each group. (A) The levels of inflammatory factors TNF- $\alpha$, IL-6 and IL-1 $\beta$, and anti-inflammatory factor IL-10 in the circulatory system of rats in each group were determined by ELISA. (B) The activity of nNOS in hippocampus tissue was determined by the enzyme biocycle method. (C) The expression of nNOS in hippocampus tissue was determined by immunohistochemical staining. Data are expressed as the mean $\pm \mathrm{SD}(\mathrm{n}=10)$. The arrows in the figure indicate the $\mathrm{nNOS}$ positive area. $\mathrm{P}<0.05 \mathrm{vs}$. sham; ${ }^{\#} \mathrm{P}<0.05$ vs. the surgery group; ${ }^{\wedge} \mathrm{P}<0.05$ vs. the surgery $+\mathrm{L}-\mathrm{NAME}$ group; ${ }^{\circledR} \mathrm{P}<0.05$ vs. the surgery + DEX group. NOS, nitric oxide synthase; nNOS, neuronal nitric oxide synthase; L-NAME, G-nitro-L-arginine methyl ester; DEX, dexmedetomidine.

T cells in the periphery, which represented the overall immune level of T lymphocyte subsets (22). Analyses of the T lymphocyte subsets demonstrated that the $\mathrm{CD}^{+} \mathrm{T}$ cell and $\mathrm{CD}^{+} / \mathrm{CD}^{+}$ $\mathrm{T}$ cell prevalence of the surgery group was lower than that of the sham group. After treatment with $\mathrm{DEX}$, the $\mathrm{CD}^{+} \mathrm{T}$ cell prevalence and $\mathrm{CD}^{+}{ }^{+} \mathrm{CD} 4^{+} \mathrm{T}$ cell percentage were significantly inverted, as demonstrated by the higher $\mathrm{CD}^{+} \mathrm{T}$ level and $\mathrm{CD}^{+} \mathrm{CD}^{+} \mathrm{T}$ cell percentage in the surgery $+\mathrm{DEX}$ group when compared with the surgery group, while surgery + DEX + L-NAME group also indicated higher figures of $\mathrm{CD}^{+} \mathrm{T}$ level and $\mathrm{CD}^{+} \mathrm{CD}^{+} \mathrm{T}$ cell percentage compared with the surgery + L-NAME group (Fig. 6A-D). Regarding the $\mathrm{CD}^{+}{ }^{+} \mathrm{CD} 8^{+} \mathrm{T}$ cell percentage, the surgery group had a significant increase in all groups except the sham group (Fig. 6E and F). In addition, the surgery + DEX + L-NAME group demonstrated a decreased tendency when compared with the surgery + L-NAME group.

\section{Discussion}

According to previous reports, there is a close association between neuro-inflammation and cognitive dysfunction caused by surgery (23-25). In the present study, cognitive function was assessed using two methods: The OFT, which has been used to detect the locomotor activity of rats (26); and the MWM test, which is a classical method to evaluate spatial learning and memory (27). The results of the present study demonstrated that surgery had no effect on the locomotor activity of rats. Compared with the sham group, the escape latency of the surgery group was prolonged and the times of crossing the platform were reduced, indicating that surgery caused cognitive impairment in aged rats and that a successful POCD model had been established. After rats were treated with L-NAME, their learning and memory functions were impaired. To hypothesize, this may be associated with the suppression of nNOS expression. However, after treatment with DEX or DEX + L-NAME, cognitive impairment was ameliorated indicating that treatment with DEX partially improved cognitive impairment induced by surgery. Previous reports have also indicated that DEX protects the cognitive impairment of surgery $(13,28)$. The present study confirmed that the use of L-NAME, an NOS inhibitor, impaired brain learning and memory function, similar to previous studies (29-31).

Neuronal apoptosis is an important cause of POCD. Previous studies have demonstrated that DEX attenuates 

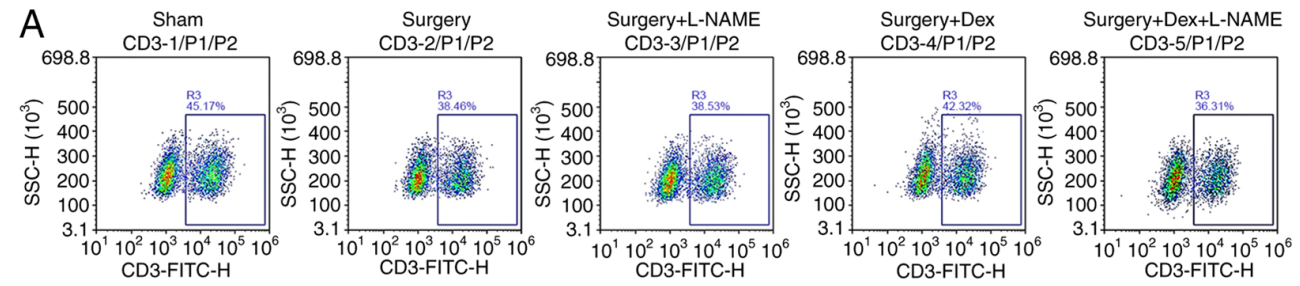

C
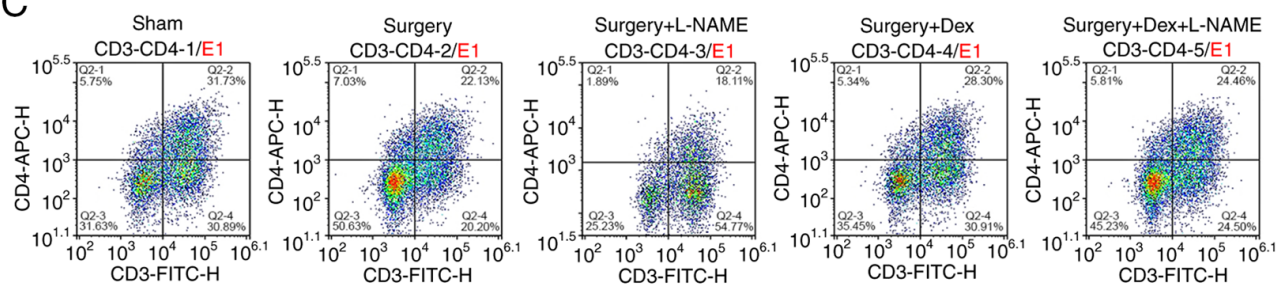

E
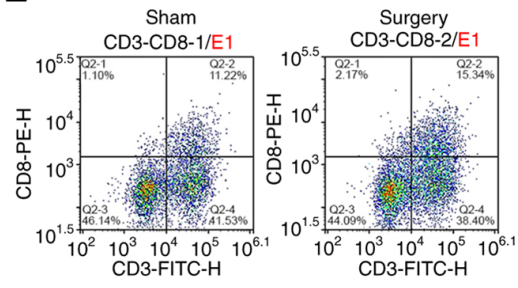
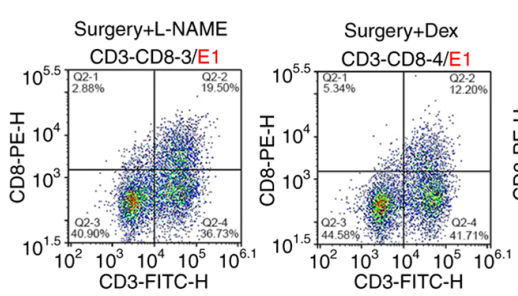

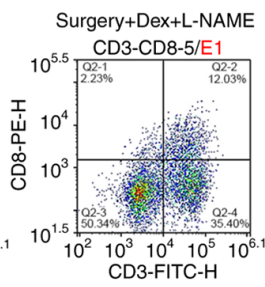

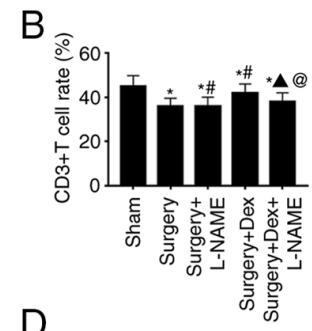
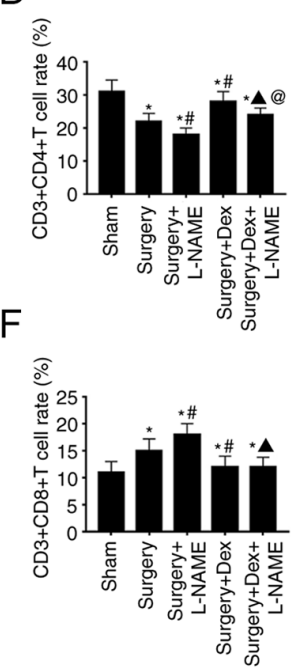

Figure 6. Flow cytometry was used to determine the T lymphocyte subsets in each group. (A) Flow cytometry dot plots of CD3 ${ }^{+} \mathrm{T}$ cell and (B) percentage of $\mathrm{CD}^{+} \mathrm{T}$ cell in each group. (C) Flow cytometry dot plots of $\mathrm{CD}^{+} \mathrm{CD}^{+} \mathrm{T}$ cell and (D) the percentage of $\mathrm{CD} 3^{+} \mathrm{CD} 4^{+} \mathrm{T}$ cell in each group. (E) Flow cytometry dot plots of $\mathrm{CD}^{+} \mathrm{CD}^{+} \mathrm{T}$ cell and $(\mathrm{F})$ the percentage of $\mathrm{CD}^{+} \mathrm{CD} 8^{+} \mathrm{T}$ cell in each group. Data are expressed as the mean $\pm \mathrm{SD}(\mathrm{n}=10)$. ${ }^{*} \mathrm{P}<0.05 \mathrm{vs}$. sham; ${ }^{\#} \mathrm{P}<0.05$ vs. the surgery group; ${ }^{\wedge} \mathrm{P}<0.05$ vs. the surgery $+\mathrm{L}-\mathrm{NAME}$ group; ${ }^{\circledR} \mathrm{P}<0.05$ vs. the surgery $+\mathrm{DEX}$ group. L-NAME, G-nitro-L-arginine methyl ester; DEX, dexmedetomidine.

neuronal apoptosis caused by isoflurane in newborn mice and reduces the occurrence of POCD $(32,33)$. TUNEL staining and western blotting were therefore conducted in the present study to determine the expression of apoptosis proteins in the hippocampus. The results demonstrated that the surgery + DEX group had a reduced apoptosis level in the hippocampus and reduced cognitive impairment compared with the sham group. The results further confirmed that surgical trauma leading to postoperative learning and memory dysfunction in aged rats was associated with neuronal damage and neuronal apoptosis in the hippocampus. Administration of DEX also reduces apoptosis in the hippocampus and reduces cognitive impairment.

Increased proinflammatory cytokines in the hippocampus may be a reason for cognitive decline following surgery (34-36). The release of inflammatory cytokines in the hippocampus may interfere with cognitive function. Normally, surgery activates the body's immune response, thereby releasing inflammatory factors. In the present study, proinflammatory cytokines in the hippocampus significantly increased on the 4th day after surgery, but returned to baseline after 1 week. The expression of proinflammatory cytokines and anti-inflammatory cytokines were also determined. The results demonstrated that the proinflammatory cytokines of the surgery group were increased, suggesting that surgery caused the inflammatory response. At 9 days after surgery, proinflammatory cytokines were decreased and anti-inflammatory cytokines were increased in the DEX and DEX + L-NAME groups.

nNOS is a constitutive neuronal enzyme that is important in regulating central nervous system function (20). A previous study demonstrated that decreased expression of nNOS in the hippocampus of POCD rats was closely associated with cognitive impairment (37). When the expression of nNOS was measured in the hippocampus in the present study, DEX was found to upregulate the expression of nNOS. However, the DEX + L-NAME group had a lower nNOS expression. Previous studies have indicated that inhibiting the expression of nNOS damages learning and memory functions (16). It is therefore hypothesized that DEX improves neuroinflammation and cognitive decline by promoting the expression of nNOS and this beneficial effect is reversed by L-NAME.

Finally, the efficiency and specificity of T lymphocytes were monitored by flow cytometry analyses. The immune response is the main cause of inflammation in the central nervous system. $\mathrm{CD}^{+} \mathrm{CD} 4^{+} \mathrm{T}$ cells can assist other related cells to participate in the immune response. Additionally, $\mathrm{CD}^{+} \mathrm{CD}^{+} \mathrm{T}$ cells are immunosuppressive and suppress the function of other immune cells (38). Previous reports have demonstrated that immune cells, especially $\mathrm{T}$ cells, have an important role in maintaining brain function, including psychological response, spatial learning and memory functions $(39,40)$. In the present study, T helper lymphocyte cells in the DEX group were higher than those of the DEX + L-NAME group, suggesting that DEX improved the immune function of rats following surgery by promoting the expression of nNOS.

In the present study, rats were intraperitoneally injected with $12 \mu \mathrm{g} / \mathrm{kg}$ DEX $30 \mathrm{~min}$ before surgery. This specific amount was used due to DEX playing a calming and analgesic function in the central nervous system, with pre-treatment 
alleviating postoperative cognitive dysfunction by inhibiting neuron excitation in aged rats (12). Thus, DEX has the potential to inhibit pathogenesis in the occurrence of cognitive dysfunction. In previous studies, DEX exerted both preconditioning and postconditioning effects against ischemic injury $(41,42)$ and the current study further determined the neuroprotective effect of DEX in preconditioning. However, the present research has several limitations. For example, experimental observations only lasted for 9 days, multiple administrations of DEX were not performed and higher doses of DEX were not administered. Therefore, further experiments are required for the application of this study in clinical therapy.

In summary, DEX played a neuroprotective role by promoting the expression of nNOS, thus inhibiting the systemic inflammatory response. This ensured a stable number of T lymphocyte subsets, thereby reducing neuronal apoptosis and reducing the occurrence of postoperative neuroinflammation.

\section{Acknowledgements}

Not applicable.

\section{Funding}

No funding was received.

\section{Availability of data and materials}

The datasets used and/or analyzed during the current study are available from the corresponding author on reasonable request.

\section{Authors' contributions}

LS conceived and supervised the study. MW was responsible for acquisition of data, analysis and interpretation of data, carried out the experiments and wrote the manuscript. LS and MW confirm the authenticity of all the raw data. All authors have read and approved the final manuscript.

\section{Ethics approval and consent to participate}

All protocols followed the requirements of the Animal Experiment Center of the Institute of Radiation Medicine of the Chinese Academy of Medical Sciences.

\section{Patient consent for publication}

Not applicable.

\section{Competing interests}

The authors declare that they have no competing interests.

\section{References}

1. Kotekar N, Kuruvilla CS and Murthy V: Post-operative cognitive dysfunction in the elderly: A prospective clinical study. Indian J Anaesth 58: 263-268, 2014.

2. Silbert B, Evered L and Scott DA: Cognitive decline in the elderly: Is anaesthesia implicated? Baillieres Best Pract Res Clin Anaesthesiol 25: 379-393, 2011.
3. Monk TG, Weldon BC, Garvan CW, Dede DE, van der Aa MT, Heilman KM and Gravenstein JS: Predictors of cognitive dysfunction after major noncardiac surgery. Anesthesiology 108: 18-30, 2008.

4. Steinmetz J, Christensen KB, Lund T, Lohse N and Rasmussen LS; ISPOCD Group: Long-term consequences of postoperative cognitive dysfunction. Anesthesiology 110: 548-555, 2009.

5. Hua M and Min J: Postoperative cognitive dysfunction and the protective effects of enriched environment: A systematic review. Neurodegener Dis 20: 113-122, 2020.

6. Wang W, Zhang XY, Feng ZG, Wang DX, Zhang H, Sui B, Zhang YY, Zhao WX, Fu Q, Xu ZP, et al: Overexpression of phosphodiesterase-4 subtypes involved in surgery-induced neuroinflammation and cognitive dysfunction in mice. Brain Res Bull 130: 274-282, 2017.

7. Lin F, Shan W, Zheng Y, Pan L and Zuo Z: Toll-like receptor 2 activation and up-regulation by high mobility group box-1 contribute to post-operative neuroinflammation and cognitive dysfunction in mice. J Neurochem: Apr 19, 2021 (Epub ahead of print).

8. Liu Q, Sun YM, Huang H, Chen C, Wan J, Ma LH, Sun YY, Miao $\mathrm{HH}$ and Wu YQ: Sirtuin 3 protects against anesthesia/ surgery-induced cognitive decline in aged mice by suppressing hippocampal neuroinflammation. J Neuroinflammation 18: 41, 2021.

9. Yuki K: The immunomodulatory mechanism of dexmedetomidine. Int Immunopharmacol 97: 107709, 2021.

10. Zhu Y, Li S, Liu J, Wen Q, Yu J, Yu L and Xie K: Role of JNK signaling pathway in dexmedetomidine post-conditioning-induced reduction of the inflammatory response and autophagy effect of focal cerebral ischemia reperfusion injury in rats. Inflammation 42: 2181-2191, 2019.

11. Zhai M, Liu C, Li Y, Zhang P, Yu Z, Zhu H, Zhang L, Zhang Q, Wang $\mathbf{J}$ and Wang J: Dexmedetomidine inhibits neuronal apoptosis by inducing Sigma-1 receptor signaling in cerebral ischemia-reperfusion injury. Aging (Albany NY) 11: 9556-9568, 2019.

12. Xiong B, Shi Q and Fang H: Dexmedetomidine alleviates postoperative cognitive dysfunction by inhibiting neuron excitation in aged rats. Am J Transl Res 8: 70-80, 2016.

13. Qian XL, Zhang W, Liu MZ, Zhou YB, Zhang JM, Han L, Peng YM, Jiang JH and Wang QD: Dexmedetomidine improves early postoperative cognitive dysfunction in aged mice. Eur J Pharmacol 746: 206-212, 2015.

14. Ge Y, Li Q, Nie Y, Gao J, Luo K, Fang X and Wang C: Dexmedetomidine improves cognition after carotid endarterectomy by inhibiting cerebral inflammation and enhancing brain-derived neurotrophic factor expression. J Int Med Res 47: 2471-2482, 2019

15. Kelley JB, Balda MA, Anderson KL and Itzhak Y: Impairments in fear conditioning in mice lacking the nNOS gene. Learn Mem 16: 371-378, 2009.

16. Zhou L and Zhu DY: Neuronal nitric oxide synthase: Structure, subcellular localization, regulation, and clinical implications. Nitric Oxide 20: 223-230, 2009.

17. Xiong B, Shi QQ and Miao CH: Dexmedetomidine renders a brain protection on hippocampal formation through inhibition of nNOS-NO signalling in endotoxin-induced shock rats. Brain Inj 28: 1003-1008, 2014

18. Zhao Z, Ren Y, Jiang H and Huang Y: Dexmedetomidine inhibits the PSD95-NMDA receptor interaction to promote functional recovery following traumatic brain injury. Exp Ther Med 21: 4, 2021.

19. Vorhees CV and Williams MT: Morris water maze: Procedures for assessing spatial and related forms of learning and memory. Nat Protoc 1: 848-858, 2006.

20. Chi MM, Lowry CV and Lowry OH: An improved enzymatic cycle for nicotinamide-adenine dinucleotide phosphate. Anal Biochem 89: 119-129, 1978.

21. Xu T, Liu J, Li XR, Yu Y, Luo X, Zheng X, Cheng Y, Yu PQ and Liu Y: The mTOR/NF- $\kappa$ B pathway mediates neuroinflammation and synaptic plasticity in diabetic encephalopathy. Mol Neurobiol: Apr 15, 2021 (Epub ahead of print).

22. Zhai S, Xu M,Li Q, Guo K, Chen H,Kong MG and Xia Y: Successful treatment of vitiligo with cold atmospheric plasma-activated hydrogel. J Invest Dermatol: May 21, 2021 (Epub ahead of print).

23. Shen X, Dong Y, Xu Z, Wang H, Miao C, Soriano SG, Sun D, Baxter MG, Zhang Y and Xie Z: Selective anesthesia-induced neuroinflammation in developing mouse brain and cognitive impairment. Anesthesiology 118: 502-515, 2013. 
24. Lin D, Cao L, Wang Z, Li J, Washington JM and Zuo Z: Lidocaine attenuates cognitive impairment after isoflurane anesthesia in old rats. Behav Brain Res 228: 319-327, 2012.

25. Cao L, Li L, Lin D and Zuo Z: Isoflurane induces learning impairment that is mediated by interleukin $1 \beta$ in rodents. PLoS One 7: e51431, 2012

26. Amin SN, Hassan SS, Khashaba AS, Youakim MF, Latif NS, Rashed LA and Yassa HD: Hippocampal and Cerebellar Changes in Acute Restraint Stress and the Impact of Pretreatment with Ceftriaxone. Brain Sci 10: 193, 2020.

27. Barnhart CD, Yang D and Lein PJ: Using the Morris water maze to assess spatial learning and memory in weanling mice. PLoS One 10: e0124521, 2015.

28. Wang WX, Wu Q, Liang SS, Zhang XK, Hu Q, Chen QH, Huang HJ, Xu L and Lou FQ: Dexmedetomidine promotes the recovery of neurogenesis in aged mouse with postoperative cognitive dysfunction. Neurosci Lett 677: 110-116, 2018.

29. Lai AY, Joo IL, Trivedi AU, Dorr A, Hill ME, Stefanovic B and McLaurin J: Cerebrovascular damage after midlife transient hypertension in non-transgenic and Alzheimer's disease rats. Brain Res 1758: 147369, 2021.

30. Bingor A, Haham T, Thornton C, Stern-Bach Y and Yaka R: Zeta inhibitory peptide attenuates learning and memory by inducing NO-mediated downregulation of AMPA receptors. Nat Commun 11: 3688, 2020.

31. Ijomone OK, Shallie PD and Naicker T: Oligodendrocytes death induced sensorimotor and cognitive deficit in N-nitro-L-arginine methyl rat model of pre-eclampsia. Neurochem Res 45: 902-914, 2020.

32. Engelhard K, Werner C, Eberspächer E, Bachl M, Blobner M, Hildt E, Hutzler P and Kochs E: The effect of the alpha 2-agonist dexmedetomidine and the $\mathrm{N}$-methyl-D-aspartate antagonist $\mathrm{S}(+)$-ketamine on the expression of apoptosis-regulating proteins after incomplete cerebral ischemia and reperfusion in rats. Anesth Analg 96: 524-531, 2003.

33. Sato K, Kimura T, Nishikawa T, Tobe $Y$ and Masaki $Y$ : Neuroprotective effects of a combination of dexmedetomidine and hypothermia after incomplete cerebral ischemia in rats. Acta Anaesthesiol Scand 54: 377-382, 2010.
34. Cibelli M,Fidalgo AR, TerrandoN,MaD,Monaco C,Feldmann M, Takata M, Lever IJ, Nanchahal J, Fanselow MS, et al: Role of interleukin-1beta in postoperative cognitive dysfunction. Ann Neurol 68: 360-368, 2010

35. Zhang J, Tan H, Jiang W and Zuo Z: The choice of general anesthetics may not affect neuroinflammation and impairment of learning and memory after surgery in elderly rats.J Neuroimmune Pharmacol 10: 179-189, 2015.

36. Zhang J, Jiang W and Zuo Z: Pyrrolidine dithiocarbamate attenuates surgery-induced neuroinflammation and cognitive dysfunction possibly via inhibition of nuclear factor $\kappa \mathrm{B}$. Neuroscience 261: 1-10, 2014.

37. Yan XB, Ouyang W, Li G and Duan KM: Involvement of neuronal nitric oxide synthase in cognitive impairment in isoflurane-treated rats. Neurosci Lett 506: 240-244, 2012.

38. Cheng DH, Liu Y and Wang L: antitumor effects of ethanol extract from ventilago leiocarpa benth on sarcoma 180 tumor-bearing mice and possible immune mechanism. Chin J Integr Med: Jan 30, 2021 (Epub ahead of print).

39. Kipnis J, Gadani S and Derecki NC: Pro-cognitive properties of T cells. Nat Rev Immunol 12: 663-669, 2012.

40. Brynskikh A, Warren T, Zhu J and Kipnis J: Adaptive immunity affects learning behavior in mice. Brain Behav Immun 22: 861-869, 2008

41. Deng Y, Cai L, Wang F, Huang J, Wang H, Li L and Lv H: Upregulated microRNA-381-5p strengthens the effect of dexmedetomidine preconditioning to protect against myocardial ischemia-reperfusion injury in mouse models by inhibiting CHI3L1. Int Immunopharmacol 92: 107326, 2021.

42. Li Y, Qu M, Xing F, Li H, Cheng D, Xing N and Zhang W The protective mechanism of dexmedetomidine in regulating Atg14L-Beclin1-Vps34 complex against myocardial ischemia-reperfusion injury. J Cardiovasc Transl Res: Apr 29, 2021 (Epub ahead of print). International (CC BY-NC-ND 4.0) License. 